\title{
REKTIONSKOMPOSITA IM DEUTSCHEN UND IM CHINESISCHEN
}

\author{
ABSTRACT: Synthetic compounds in German and Chinese
}

The subject of this article are nominal compounds in German and Chinese which are assigned to the class of synthetic compounds. Such compounds are based on a verb stem, to which are added lexical morphemes and/or suffixes anchored in the grammar and semantics of the (verbal) head. In German, the verb with the suffix forms a derivative which, as the basic word, selects an argument (object or subject) in function of the determinative word. In this way, a government relationship is established between the constituents of the compound. This applies equally to action nouns and agent nouns. In Chinese, there are not formal exponents of the action noun, so the analysis had to be limited to agent nouns. In their derivation, the verb stem and its object mostly form a constituent that acts as a complement of the agent exponent. The examination of such synthetic compounds is carried out from the perspective of the argument linking.

KEYWORDS: synthetic compound, argument linking, German, Chinese, word formation

\section{Einleitende Bemerkungen ${ }^{1}$}

Deutsch und Chinesisch ${ }^{2}$ weisen in der Wortbildung, v. a. in der Zusammensetzung, trotz ihrer genetischen und typologischen Entfernung bestimmte Ähnlichkeiten auf. Nach Greenberg (1963, S. 92.) existieren keine Sprachen ohne Komposition und Derivation, d. h., es gibt keine rein isolierenden Sprachen. Die Anzahl der flexionslosen Sprachen sei zwar beachtlich, aber höchstwahrscheinlich gebe es keine ohne Komposition und Derivation, obwohl mittlerweile festgestellt wurde, dass es marginal Sprachen ohne Komposition wie das Westgrönländische gibt (vgl. Scalise \& Vogel, 2010, S. 1). Deutsch wird den flektierenden Sprachen zugeordnet, dennoch besitzt es u. a. in der Wortbildung einen bedeutenden Teil der Analyse. Und gerade hier lassen sich

\footnotetext{
Yaqing Yang - Sichuan International Studies University, Chongqing, yyqsisu@163.com

${ }^{1}$ Meinem Kollegen Jaroslaw Aptacy danke ich für hilfreiche Bemerkungen und die Korrektur des Manuskripts.

${ }^{2}$ Mit Chinesisch ist hier das Mandarin gemeint.
} 
gemeinsame Punkte mit Chinesisch erkennen: Beide Sprachen weisen endozentrische Komposita auf, in denen der Kopf die Bedeutung und die grammatischen Merkmale der ganzen Konstruktion festlegt. Auch auf Deverbativa basierende Rektionskomposita (RK, engl. synthetic compounds) sind in den beiden Sprachen festzustellen, hier bestehen jedoch Divergenzen in der Serialisierung der Konstituenten. Deutsch ist in dieser Hinsicht kopffinal, während sich Chinesisch durch eine größere Kopfstellungsvariabilität auszeichnet.

Der vorliegende Beitrag setzt sich zum Ziel, die internen Beziehungen zwischen unmittelbaren Konstituenten der RKs, v. a. zwischen Kopf und Komplement, zu untersuchen. Der Untersuchungsgegenstand umfasst die nominale Zusammensetzung (Nomina actionis und Nomina agentis im Deutschen, Nomina agentis im Chinesischen), da gerade dieser Kompositionstyp im Deutschen wie im Chinesischen am stärksten vertreten ist. Im folgenden Abschnitt soll der Begriff RK bestimmt und von anderen Komposita abgegrenzt werden.

\section{Abgrenzung der RKs}

RKs sind Komposita, bei denen ein Rektionsverhältnis zwischen dem Determinatum (Grundwort) und dem Determinandum (Bestimmungswort) vorausgesetzt wird (vgl. Eichinger, 2000, S. 128). RKs sind in dem hier interessierenden Fall nominale Komposita ${ }^{3}$, bei denen das Bestimmungsglied ,eine offene Stelle in der Argumentstruktur des Kopfs der Wortstruktur" (Olsen, 1986b, S. 67; vgl. auch Rivet, 1999, S. 307; Haspelmath \& Sims, 2010, S. 249) füllt. Es sind also Wortbildungskonstruktionen, „bei denen ein Glied eine grammatische Rektion besitzt, die zur Deutung des Kompositums ausgenutzt wird“(Olsen, 1986b, S. 66) oder schlicht „komplexe Wörter mit deverbalem Zweitglied“"(Rivet, 1999, S. 307).

Den oben angeführten Definitionen entsprechend fokussiert der vorliegende Beitrag Bildungen, deren Zweitglied (Determinatum) ein deverbales Derivat ist und die Wahl des Erstgliedes (Determinandum) determiniert. Z. B. ist die Bedeutung von Schadensbegrenzung so zu verstehen, dass Begrenzung - ähnlich wie seine Basis begrenzen genauer spezifiziert werden kann. Es ist in diesem Fall nicht irgendeine Begrenzung, sondern eine Begrenzung von Schaden/Schäden. Es ist also genannt worden, was der Gegenstand der Begrenzung ist. Das Bestimmungswort ist somit in der Bedeutung des Grundwortes verankert, aber es ist auch in dessen Grammatik vorgesehen, da $\mathrm{Be}$ grenzung als deverbales Nomen die Argumentstruktur (AS) des zugrunde liegenden Verbs begrenzen im Prozess der Nominalisierung erbt. Die Interpretation der RKs ist

\footnotetext{
${ }^{3}$ RKs werden meist am Beispiel substantivischer Komposita untersucht, aber dieser Typ beschränkt sich nicht nur auf Substantive. Auch adjektivische RKs werden analysiert (vgl. z. B. Olsen, 1986a; Schmidt, 1987; Fuhrhop, 2007; Fleischer \& Barz, 2012; Aptacy, 2015a).
} 
demnach nicht frei, sondern sie richtet sich nach der Grammatik und Semantik des deverbalen Derivats, das seinerseits die grammatischen und semantischen Merkmale der verbalen Basis erbt.

Der Typ der RKs ist nach der Nominalisierungstypologie von Toman (1983, S. 86 f.) der wort- und kompositumsinternen ${ }^{4}$ Realisierung verbaler Argumente zuzuordnen, vgl.:

$$
\text { Strom wird erzeugt } \rightarrow \text { Stromerzeugung }
$$

Die Nominalphrase Stromerzeugung repräsentiert den Typ der RKs, da das Determinandum an der Sättigung der AS des Determinatums beteiligt ist, in diesem Fall fungiert das Nomen Strom als Objektargument zu Erzeugung. Dies ist ein durchaus produktives Wortbildungsmuster im Deutschen, nach dem ständig neue Konstruktionen gebildet werden (vgl. Kürschner, 1974, S. 190).

Die RKs werden von Nichtrektionskomposita abgegrenzt, bei denen das Bestimmungswort nicht in Grammatik und Bedeutung des Grundworts verankert ist, ihre semantische Interpretation zeichnet sich durch zahlreiche Idiosynkrasien aus und wird erst durch den Sprachgebrauch festgelegt. Nach Fanselow (1981, S. 48), gibt es [...] die Bedeutung eines Kompositums gar nicht”, Holzauto kann als „Auto aus Holz“ oder als „Auto, mit dem Holz befördert wird“ verstanden werden. Die semantische Beziehung zwischen Erst- und Zweitglied eines Nichtrektionskompositums ist somit prinzipiell offen und lässt sich aufgrund des Wissens erschließen, das an der Grenze zwischen Semantik und Pragmatik angesiedelt ist (vgl. Vater, 2002, S. 81). Jedes Kompositum kann in mehreren Weisen interpretiert werden, von denen sich aber meistens nur eine durchsetzt.

Bezüglich der Rektionskomposita selbst kann hinterfragt werden, ob (i) alle Argumenttypen der Deverbativa am Aufbau der RKs beteiligt sind und ob (ii) auch Adjunkte / freie Angaben als Konstituenten betrachtet werden können, die im rektionellen Verhältnis zum Grundwort stehen.

Zu (i): Rivet (1999, S. 308, 317, 324) ist der Ansicht, dass nur Akkusativobjekte (AO) innerhalb eines RK auftreten könnten, da es solche Bildungen wie *Frauenschenker, *Mordbeschuldiger oder *Katharinadenker nicht gebe. In Frauenschenker entspricht das Bestimmungswort dem Dativobjekt $(\leftarrow$ jemand schenkt (den) Frauen etwas), in Mordbeschuldiger dem Genitivobjekt $(\leftarrow$ jemand beschuldigt jemanden eines Mordes) und in Katharinadenker dem Präpositionalobjekt $(\leftarrow$ jemand denkt an Katharina). Dagegen können RKs mit auf AO zurückgehenden Bestimmungswörtern nahezu uneingeschränkt gebildet werden, vgl. z. B. Lebensschützer, Schuldzuschreibung, Tatsachenverdrehung u. Ä. Dem einen strukturellen Kasus tragenden AO kommt zwar eine

\footnotetext{
${ }^{4}$ Daneben werden noch (i) wort- und kompositumsexterne Realisierung der Argumente (z. B. Die Geheimdienste überwachen das Internet. $\rightarrow$ die Überwachung des Internets durch die Geheimdienste) sowie (ii) eine Kombination aus wortinterner und wortexterner Realisierung unterschieden (z. B. Die Internetüberwachung durch die Geheimdienste).
} 
besondere Stellung unter den Verbargumenten zu (vgl. Sabel, 2002, S. 235 f.), dennoch können auch andere Objekte sowie das Subjekt mit Deverbativa Komposita bilden, so dass wohl auch in diesem Fall von RKs die Rede sein kann, vgl. in Frauenhilfe entspricht Frauen dem Dativobjekt ( $\leftarrow$ jemand hilft Frauen), in Freiheitsberaubung geht Freiheit auf ein Genitivobjekt zurück $(\leftarrow$ man hat jemanden der Freiheit beraubt), in Drogenhandel ist Drogen mit einem Präpositionalobjekt zu verbinden $(\leftarrow$ Es wird mit Drogen gehandelt) und bei Schneefall entspricht das Bestimmungswort dem Subjekt des Basisverbs fallen (vgl. Aptacy, 2015b, S. 4).

$\mathrm{Zu}$ (ii): Obwohl freie Angaben/Adjunkte als Konstituenten gelten, die nicht durch die Rektion gefordert werden, werden sie z. B. von Eichinger (2000, S. 129) auch als Bestandteile von RKs betrachtet. Seiner Ansicht nach gehören Wortbildungskonstruktionen zu „Rektionskomposita [...] nur, insoweit entsprechende Ergänzungs- und adverbiale Angaberelationen aufgenommen werden“. Daraus folgt, dass Komposita wie Sozialverhalten, Lautlesen und Getrenntschreibung als RKs betrachtet werden. Bei Sozialverhalten könnte evtl. dafür der Umstand sprechen, dass sozial bei Verhalten eine obligatorische Ergänzung ist (vgl. *Er verhält sich. vs. Er verhält sich sozial.), was jedoch nicht auf laut bei Lesen und getrennt bei Schreibung bezogen werden kann, da sie als fakultative Angaben nicht in der AS der Regentien verankert sind.

In diesem Aufsatz wird ein Mittelweg zwischen der Auffassung von Rivet und der von Eichinger beschritten. Es wird somit angenommen, dass am Aufbau der RKs alle Argumente beteiligt sind, soweit sie in der AS des Kopfes vorgesehen sind, d. h. alle Objekte und Subjekte, andererseits werden adverbiale Relationen zwischen Konstituenten bei der Analyse der RKs ausgeschlossen.

\section{Argumentstruktur der RKs im Deutschen}

Da im Mittelpunkt dieses Beitrags die Argumentvererbung in den RKs steht, wird im folgenden Abschnitt ihre AS präsentiert. Relationale lexikalische Einheiten besitzen Argumentstellen, die bei der Einführung in Strukturen gefüllt werden müssen, unabhängig davon, ob es sich um syntaktische oder lexikalische Strukturen/ Bäume handelt (vgl. Lieber, 1983, S. 251). Folgerichtig wird nach der Beschreibung der ASs ein denkbarer Mechanismus präsentiert, der die Argumentrealisierung in den RKs erklärt.

Das Untersuchungsmaterial umfasst ausschließlich deverbale Ableitungen, welche die semantischen Klassen der Nomina actionis und Nomina agentis/instrumenti vertreten. Als Kopf solcher Ableitungen wird das Suffix betrachtet, das die Fähigkeit besitzt, die morphosyntaktische Kategorie und die grammatischen Merkmale des ganzen Derivats festzulegen. Ansonsten ist in dem suffixalen Lexikoneintrag die Kategorie des Komplements bestimmt, welches das Suffix selegiert. Es kann jedoch keine Thetarollen 
zuweisen, weil es keine AS besitzt. Diese erbt das Suffix vom Komplement, mit dem es sich verbindet (vgl. Szigeti, 2002, S. 10; Vater, 2002, S. 80). Beispielsweise kann für das bei Nomina actionis produktivste Suffix -ung der Lexikoneintrag wie folgt dargestellt werden:

$$
\text { /ung/; [-V, +N, +Fem], SK [V_] }
$$

Im Eintrag (2) sind folgende Informationen enthalten: (i) das Suffix leitet Nomina $[-\mathrm{V},+\mathrm{N}] \mathrm{ab}$, (ii) es legt das feminine Genus der Ableitungen [+Fem] fest und (iii) es verbindet sich vornehmlich mit verbalen Stämmen, d. h., seine Subkategorisierung ist [ $\mathrm{V}_{-}$] (Information über das Komplement). Dies soll verhindern, dass adnominale ${ }^{5}$ oder deadjektivische Derivate wie *Tischung oder*Schnellung entstehen, dafür aber zahlreiche Deverbativa wie Empörung oder Beerdigung. Bei ihrer Entstehung vereinigt sich das Suffix mit dem lexikalischen verbalen Komplement zu einer neuen Einheit. Diese verfügt über eine eigene AS, die sie vom Komplement erbt:

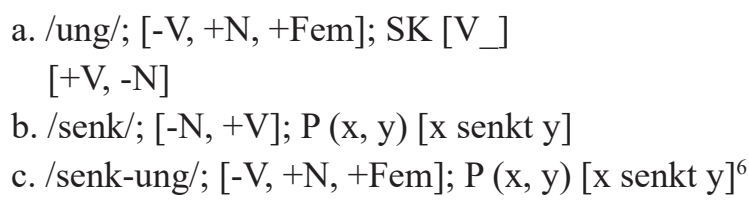

Aus (3)c. folgt, dass Senkung Wortklasse und Genus vom Suffix bekommt, die transitive AS dagegen wird vom Stamm senk aus (3)b geerbt. Das Derivat erhält also einen bedeutenden Teil seiner grammatischen Merkmale von der Basis, was z. B. Selkirk (1982, S. 39f.) folgendermaßen auf den Punkt bringt: „A very regular relation holds between the lexical form of a verb and the lexical form of an affixed constituent built on the base of that verb".

Ähnlich kann das Suffix -er für deverbale Nomina agentis/instrumenti charakterisiert werden, im Unterschied zu -ung bestimmt es aber das maskuline Genus des Derivats. Die Agensbedeutung von -er hat ansonsten die Konsequenz, dass es die Realisierung des Subjekts verhindert, das eigentlich in -er schon enthalten ist ${ }^{7}$ :

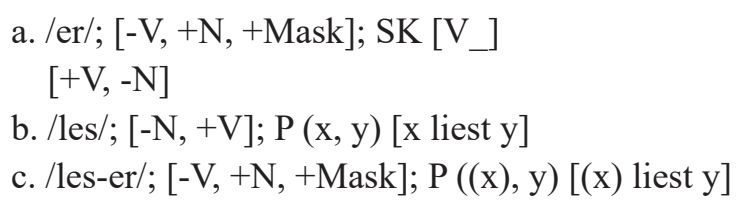

${ }^{5}$ Sporadisch kann man auch adnominale oder deadjektivische $u n g$-Derivate wie Hausung oder Rundung begegnen. Sie treten jedoch vereinzelt auf, so dass sie als festgeprägte Lexikoneinheiten zu betrachten sind und nicht im System produktiver Wortbildungsmuster berücksichtigt werden müssen.

${ }^{6}$ Leicht abgewandelt nach Bierwisch (1989, S. 13).

${ }^{7}$ Dies betrifft nicht Nomina acti auf -er wie z. B. Seufzer der Mutter oder Versprecher des Ministerpräsidenten, vgl. Vater (2002, S. 80). 
Die Argumentvererbung ist mit der bei ung-Derivaten vergleichbar, der einzige Unterschied besteht darin, dass das Subjektargument $x$ in (4)c. im Wort selbst realisiert wird. Die runde Klammer $(x)$ soll dies zum Ausdruck bringen. Diese Blockierung vermag auch zu erklären, warum das lexikalische Subjekt überhaupt nicht erscheinen kann, sei es im Derivat oder außerhalb, vgl.
a. $\rightarrow$ Die Eltern unterstützen ihre Kinder.
b. $\rightarrow$ die Unterstützer der Kinder
c. $\rightarrow *$ die Kinderunterstützer durch die Eltern
d. $\rightarrow *$ die Elternkinderunterstützer

Nach (4)a. ist -er für verbale Basen subkategorisiert, aber es kann auch an nominale Stämme angehängt werden. Solche Ableitungen treten jedoch viel seltener auf und sind keine RKs, vgl.: Berliner, Eisenbahner, Fischer, Fußballer, Gewerkschafter, Musiker, Straßenbahner.

\section{Mechanismen der Argumentvererbung in deutschen RKs}

Im Mittelpunkt dieses Abschnitts steht die Argumentvererbung in RKs, die den Nomina actionis auf -ung und Nomina agentis auf $-e r^{8}$ zugeordnet werden.

Die Analyse beschränkt sich auf RKs, die auf transitive Verben zurückgehen. In solchen Bildungen korreliert das Bestimmungswort am häufigsten mit dem AO des Basisverbs, z. B. Menschenrechtsverletz-ung, Steuervermeid-ung, Geschlechtsneutralisier-ung, Wojtyla-Verehr-ung. Neben -ung können auch andere Suffixe deverbale Nomina actionis ableiten, auch wenn -ung in dieser Funktion am häufigsten auftritt: -e (Minensuch-e, Amtsübernahm-e), -ion (Fleischprodukt-ion, Bilanzmanipulat-ion). In diese Gruppe gehören auch suffixlose implizite Ableitungen/Konversionen (Rechtsbruch, Siemens-Umbau). Nomina agentis/instrumenti werden meistens mit -er erzeugt (Erdgashändl-er, Ausbildungsabbrech-er), seltener mit -ent (Fleischproduz-ent) oder -ant (Stromliefer-ant).

Wegen ihrer Überschaubarkeit eignen sich zur Darstellung der Argumentvererbung besonders die Strukturbäume. Zunächst wird dieser Mechanismus an einem Nomen actionis erklärt, woraufhin zu Nomina agentis/instrumenti übergegangen wird. Beispielsweise sieht das Baumdiagramm für Konzernumbenennung folgendermaßen aus:

${ }^{8}$ Mit diesem Suffix werden auch Nomina instrumenti gebildet, so dass nicht immer eindeutig ist, welcher Typ gerade vorliegt (z. B. Lastenträger). Erst der Sprachgebrauch entscheidet, ob sich die eine oder andere Lesart durchsetzt. 
(6) KonzernumbenennungN ${ }^{0}[-\mathrm{V},+\mathrm{N},+\mathrm{Fem}]$, [Ag-Sub, Th-Ob]
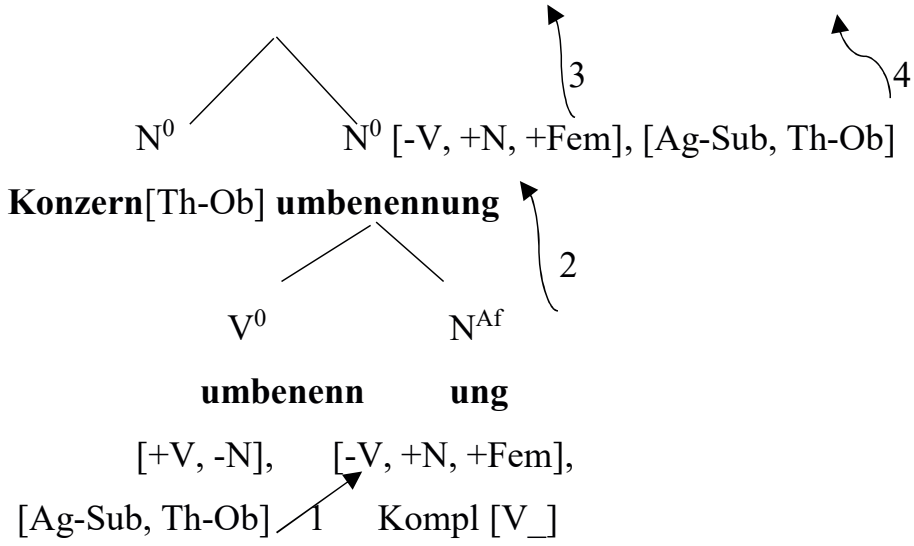

Die grammatischen Merkmale des Suffixes werden entlang der Projektionslinie auf das ganze Derivat übertragen (Pfeile 2 und 3), was die Bestimmung der Wortart und der grammatischen Kategorien des RK durch das Suffix zum Ausdruck bringt. Die thematischen Rollen des [+V]-Komplements, Agens und Thema, werden an das Suffix weitergeleitet (Pfeil 1); die Rollen werden syntaktisch als Subjekt (Ag-Sub) und Objekt (Th-Ob) kodiert. Das Ergebnis der Verkettung von umbenenn und -ung ist umbenennung, das ein Nomen ist und die vom Verb geerbten Argumente Agens sowie Thema besitzt. Wenn ein Komplement wie Konzern hinzugefügt wird, erhält es die Rolle Thema, damit wird das Thema-Argument von umbenennung abgebunden. Werden die thematischen Merkmale von Konzern und umbenennung abgeglichen, werden sie bei dem letzteren getilgt, was die Durchstreichung an der Baumwurzel symbolisiert. Das Subjektargument kann kompositumsextern als durch-Phrase auftreten (vgl. Anm. 4), z. B. in Konzernumbenennung durch die Aktionäre. Auf diese Weise werden bspw. RKs wie Fleischfertigung, Patentanmeldung, Finanzoptimierung u. a. m. generiert.

Die Argumentvererbung in einem Nomen agentis/instrumenti soll das folgende Diagramm für Tierschützer schildern:

$$
\text { Tierschützer } \mathrm{N}^{0}[-\mathrm{V},+\mathrm{N},+\mathrm{Mask}] \text {, [Th-Ob] }
$$

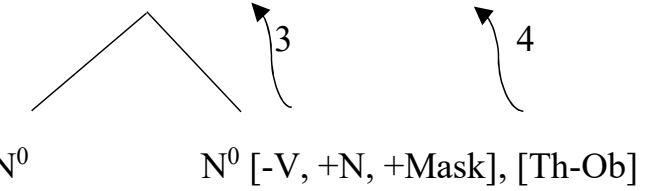

Tier $[\mathrm{Th}-\mathrm{Ob}]$

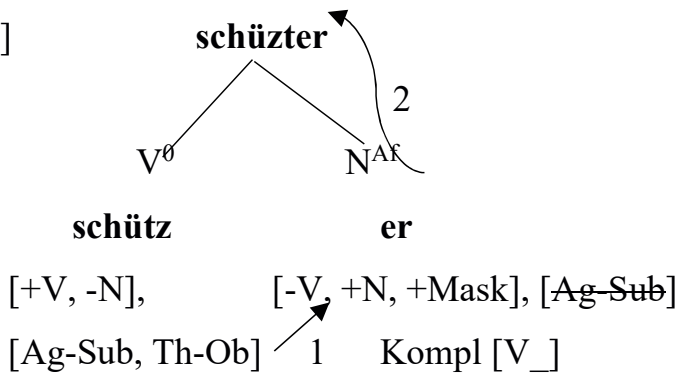


Die Argumentvererbung in (7) sieht ähnlich wie in (6) aus, jedoch mit dem Unterschied, dass bei der Bildung der Nomina agentis die Subjekt-Argumentstelle bereits auf der Etappe der Suffigierung abgebunden und nicht weiter projiziert wird. Das blockiert den Subjektanschluss in Form einer Präpositional- oder Nominalphrase (vgl. (5) c., d.). Leer bleibt die Stelle für das thematische Objekt, die durch eine Verbindung des er-Derivats mit einem Nomen besetzt wird (Durchstreichung oben). In diesem Fall ist es die Komposition von schützer und Tier. Weitere Beispiele für RKs, die nach diesem Muster gebildet werden: Kreditvermittler, Hähnchenmäster, Zementhersteller u. v. a. m.

\section{RKs im Chinesischen}

\subsection{Allgemeines}

Das Vorhandensein der RKs im Chinesischen wurde verhältnismäßig früh erkannt, denn schon in den 1940er Jahren wies der chinesische Linguist Lu Shuxiang (1942, zit. nach Gu/Shen, 2001, S. 123) auf diesen besonderen Wortbildungstyp hin. Die diesem Typ folgenden Wörter weisen ein Agens und ein Patiens auf, was sie in die Nähe des Satzes rückt. Dabei werden sie in vier Typen eingeteilt:

I. 旅客 (lu ke-,Reisende(r) ${ }^{\natural}$ ), 舞女 (wu nü-,Tanzmädchen ‘9), 不倒翁 (budao weng - ,Stehaufmännchen'), 未亡人 (weiwang ren - ,Witwe' (als Selbstbezeichnung)),

II. 牧童 (mu tong -,Hirtenjunge'), 搭客 (dake-,Fahrgast'), 研究员 (yanjiu yuan - ,Forscher'),

III. 售票员 (shou piao yuan -,Schaffner'), 编剧人 (bian ju ren -,Drehbuchautor'), 浣纱女 (huan sha nü-,Wäscherin'), 食蚁兽 (shi yi shou -,Ameisenbär'),

IV. 日报读者 (ribao du zhe -,Zeitungsleser'), 电影演员 (dianying yan yuan,Filmschauspieler'), 飞机乘客 (feiji cheng ke-,Fluggast').

Komposita unter I besitzen nur ein Agens, unter II auch, aber hier wurde das Patiens getilgt. In III und IV sind Agens und Patiens vorhanden, unterschiedlich ist lediglich deren Reihenfolge. Die Komposita unter III und IV würde man nach den oben fürs Deutsche formulierten Kriterien als Rektionskomposita bezeichnen. Da ihre Struktur auf der AS des Basisverbs fußt, kann hier auch von einer Argumentvererbung die Rede sein. Einer der Unterschiede besteht jedoch darin, dass im Deutschen das Suffix über die grammatische und semantische Kategorie der ganzen Konstruktion entscheidet, im Chinesischen sind es dagegen Morpheme, die auch als selbständige Lexeme auftreten können. Allerdings wird auch ihre Zuordnung zu reihenbildenden Suffixen erwogen ${ }^{10}$.

${ }^{9}$ Berufliche Bezeichnung für Frauen im 20. Jahrhundert, die in einem Ballsaal mit Männern tanzen.

${ }^{10} \mathrm{Vgl}$. 曲英梅, 基于语料库的英汉动名化研究, 吉林 [unver. Dissertation, 2009], Nordöstliche Pädagogische Universität, S. 67-69. 
Die Vererbung und Reihenfolge der Argumente in chinesischen RKs bildet mittlerweile den Gegenstand zahlreicher Untersuchungen, deren Ergebnisse u. U. bedeutend voneinander abweichen. Und so führt He (2004) eine „Right-hand Head Rule“ ein, welche die Struktur OVS erklärt; das ist der default-Fall der Konstituentenabfolge in den chinesischen RKs, im Unterschied zu Satzstrukturen, die sich durch die kanonische SVO-Linearisierung auszeichnen ${ }^{11}$.

Cheng (2005) analysiert RKs mit - 者 (zhe - Suff -er). Er glaubt, VO-者 sei die grammatisch standardisierte Form eines RKs mit - 者, wie z. B. 造谣者 (zao yao zhe, Verleumder'), wodurch er sich von He (2004) unterscheidet, der in der Morphemfolge $\mathrm{VO}(\mathrm{S})$ eher eine durch Syntax erzeugte Verbalphrase sieht, die ,,is looped back from syntax to lexicon to become a stem of a compound" ${ }^{\text {"12 }}$.

Hong \& Shi (2012, S. 78) gehen von Syntax und Prosodie aus und diskutieren ebenfalls die Kombination der Argumente in Komposita. Sie stellen fest, dass z. B. 汽 车修理工 (qiche xiuli gong - ,Automechaniker') als Kompositum über die Struktur $\left[\left[\mathrm{N}_{1}+\mathrm{V}\right]+\mathrm{N}_{2}\right]$ verfügt, indem sie die nominale Partikel 的 (de) als Testmittel einfügen. An Konstruktionen wie (i) 汽车的修理工 und (ii) *汽车修理的工 zeigen die Autoren, dass (i) $\left[\mathrm{N}_{1}+\left[\mathrm{V}+\mathrm{N}_{2}\right]\right]$ eher eine durch Syntax erzeugte phrasale Struktur ist, während (ii) $\left[\left[\mathrm{N}_{1}+\mathrm{V}\right]+\mathrm{N}_{2}\right]$ ein Wort, ein Kompositum, ist. Die Möglichkeit, nach dem $\mathrm{N}_{1}$ 汽车 in (i) die Partikel 的 einzufügen, zeugt davon, dass die Struktur nicht lexikalisiert ist und in der Syntax erzeugt wird. In (ii) dagegen, wo nach $\left[\mathrm{N}_{1}+\mathrm{V}\right]$ die Partikel nicht erscheinen kann, liegt eine lexikalisierte Bildung vor, deren interne Struktur für syntaktische Prozesse ,unsichtbar“ ist (vgl. Hong \& Shi, 2012, S. 80).

\subsection{Argumentvererbung}

Den Gegenstand des folgenden Abschnitts bilden ausgewählte Mechanismen der Argumentvererbung innerhalb der chinesischen RKs. Da für diese Sprache nicht eindeutig entschieden zu sein scheint, welche Strukturen mit (de-)verbalem Kern als Lexeme bzw. (Verbal-)Phrasen zu betrachten sind, wird für die Zwecke des vorliegenden kontrastiven Beitrags als Arbeitshypothese angenommen, dass es sich im Falle solcher Bildungen durchweg um RKs handelt. Ausschlaggebend ist hier das Kriterium der (de-)verbalen Ableitungsbasis und die Okkurrenz der Argumente des Basisverbs.

Die RKs wie 项目负责人 (xiangmu fuze ren - ,Projektleiter'), 国家领导人 (guojia lingdao ren - ,Staatschef'), 空调安装工 (kongtiao anzhuang gong - ,Klimaanlageninstallateur'), 日报读者 (ribao du zhe-,Zeitungsleser'), 电影演员 (dianying yan yuan - ,Filmschauspieler'), 飞机乘客 (feiji cheng ke-,Fluggast ${ }^{`}$ ) zeichnen sich

${ }^{11} \mathrm{http} / / /$ en.cnki.com.cn/Article_en/CJFDTOTAL-DDYX200403001.htm [Zugriff: 29.04.2018]

$12 \mathrm{http} / / /$ en.cnki.com.cn/Article_en/CJFDTOTAL-DDYX200403001.htm [Zugriff: 29.04.2018] 
durch die Abfolge OVS aus: Das Objekt erscheint links vom Basisverb, das Subjekt als Affix/Stammmorphem bildet auf der rechten Seite den Konstruktionskopf. Eine solche Linearisierung bietet ein Spiegelbild der SVO-Grundsatzgliedfolge im chinesischen Satz: 日报读者 $\rightarrow$ 者-读-日报 (ribao du zhe $\rightarrow$ zhe du ribao - ,Eine Person liest die Tageszeitung $)^{13}$. Zu bemerken ist, dass 者 als Satzsubjekt eigentlich nicht zulässig ist, es erscheint aber als Subjektargument im RK, in dem auch andere einsilbige Morpheme wie 人 (ren), 家 (jia), 员 (yuan) etc. vorkommen ${ }^{14}$. Die Wahl des einen oder anderen richtet sich nach deren Semantik und ist im Gegensatz zu einer Phrase wie 读日报的人, 读日报者, wo sie paradigmatische Beziehungen eingehen können, nicht austauschbar. Da die chinesischen Morpheme zur Ableitung der Nomina agentis semantische Unterschiede aufweisen, lassen sich damit präzisere Bedeutungen ausdrücken. Und so werden - im Vergleich zu allgemeinen 人 (ren) und 者 (zhe) Personen, die in einem Bereich besonders erfolgreich sind und zu dessen Entwicklung sie merklichen Beitrag geleistet haben, als 家 (jia) bezeichnet, 工 (gong) werden Techniker oder Handwerker genannt, 员 (yuan) ist ein in einem Bereich tätiger und kompetenter Mensch. Im Vergleich dazu hat man es im Deutschen v. a. mit dem Suffix -er zu tun, das eine sehr allgemeine Semantik besitzt. Semantische Feinheiten lassen sich in dieser Sprache kompositionell aufgrund der Bedeutungen des Verbstamms und des Objektarguments ausdrücken, während das Suffix allgemein den Handelnden bezeichnet, vgl. z. B. Ameisen-forsch-er, Steuer-hinterzieh-er, Stein-klopf-er, Fliesen-leg-er u. a. m.

Für die Darstellung der Argumentvererbung ist die Frage nach der Hierarchie unter den Konstituenten der RKs ausschlaggebend: Geklärt werden muss, über welche Zwischenstufen die Komposition erfolgt: Bildet das Basisverb eine engere Verbindung mit dem Subjekt, zu der das Objekt hinzutritt, oder es entsteht zunächst eine Konstituente aus Verb und Objekt, die als Ganzes das Komplement des Subjekts darstellt? Dementsprechend wäre Struktur (8) oder (9) anzunehmen:

$$
\begin{aligned}
& {\left[\mathrm{N}_{1}+\left[\mathrm{V}+\mathrm{N}_{2}\right]\right]_{\mathrm{RK}}} \\
& {\left[\left[\mathrm{N}_{1}+\mathrm{V}\right]+\mathrm{N}_{2}\right]_{\mathrm{RK}}}
\end{aligned}
$$

Wie oben angedeutet, überwiegt in der Literatur zu chinesischen RKs die Ansicht, dass die meisten die Struktur (9) aufweisen, d. h., wie im Deutschen geht das Objekt mit dem Verb eine engere Verbindung ein als das Subjekt (vgl. Zhuang \& Liu, 2011). Das folgende Diagramm soll - der Struktur in (9) entsprechend - die Argumentvererbung im RK 项目负责人 (xiangmu fuze ren) exemplarisch darstellen:

${ }^{13} \mathrm{http}: / /$ en.cnki.com.cn/Article_en/CJFDTOTAL-YWZG200102002.htm [Zugriff: 29.04.2018]

${ }_{14}$ Dies lässt, zumindest im Falle von 者, Satz- von lexikalischen Strukturen unterscheiden. 
(10)

项目负责人 $[-\mathrm{V},+\mathrm{N}][\mathrm{Th}-\mathrm{Ob}, \mathrm{Ag}-\mathrm{Sub}]$

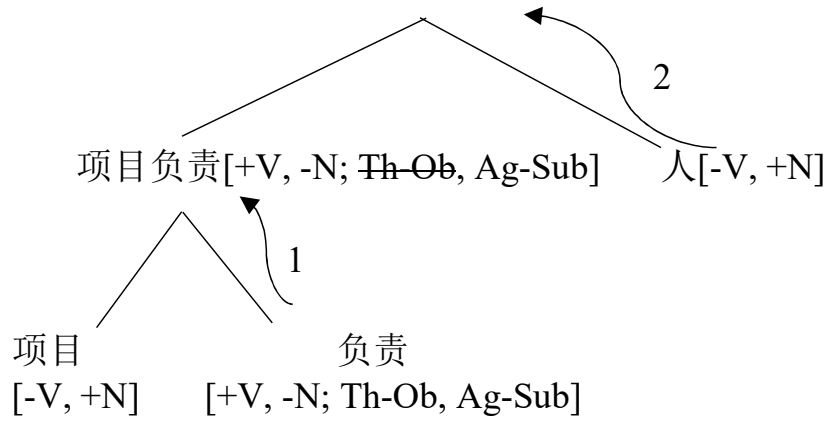

Das Verb 负责 (fuze) geht mit seinen grammatischen und thematischen Merkmalen in die Struktur ein, das Objekt项目 (xiangmu) füllt die dafür vorgesehene Argumentstelle aus, woraus die Sättigung dieser Argumentposition erfolgt (Pfeil 1 und Durchstreichung). Als Nächstes wird der nominale Kopf 人 (ren) mit der OV-Konstituente 项目负责 verkettet; dadurch wird deren Subjekt-Argumentstelle ausgefüllt und RK 项目负责人 gebildet (Pfeil 2, Durchstreichung). Nach diesem Muster werden z. B. OVS-RKs gebildet wie: 国家领导人 (guojia lingdao ren - ,Staatschef'), 空调安装 工 (kongtiao anzhuang gong -,Klimaanlageninstallateur'), 火箭发射员 (huojianfashe yuan - ,Raketenabschuss-Bedienungspersonal'), 出租车驾驶员 (chuzuche jiashi yuan - ,Taxifahrer') u. a. m.

Neben der dominierenden OVS-Stellung treten im Chinesischen auch Bildungen mit der VOS-Abfolge auf. Deren Zuordnung zu RKs ist zwar nicht unumstritten (s. o.), dennoch sollen sie hier aufgrund der Argumentrealisierung des Basisverbs als RKs betrachtet werden. Die Basis solcher Konstruktionen bilden doppelsilbige Verben und ebensolche Objekte (11) oder einsilbige Verben und einsilbige Objekte (12):

(11) 传播病毒者 (chuanbo bingdu zhe -,Virus'), 崇拜金钱者 (chongbai jinqian zhe -, Anbeter'), 开创事业者 (kaichuang shiye zhe - ,Unternehmensgründer'),

(12) 造谣者 (zaoyao zhe -,Schöpfer'), 理发师 (lifa shi-,Friseur'), 毕业生 (biye sheng - ,Absolvent'), 管家婆 (guanjiapo - ,Haushälterin').

Alle in (11) angegebenen Beispiele können auch die OVS-Abfolge realisieren:

(11) a. 病毒传播者, 金钱崇拜者, 事业开创者

Bei RKs mit einsilbigem Verb und Objekt besteht diese Möglichkeit jedoch nicht, d. h., solche Komposita zeichnen sich durch eine größere strukturelle Festigkeit aus, weswegen ein Beispiel aus dieser Gruppe die Argumentvererbung in der VOS-Struktur präsentieren soll: 


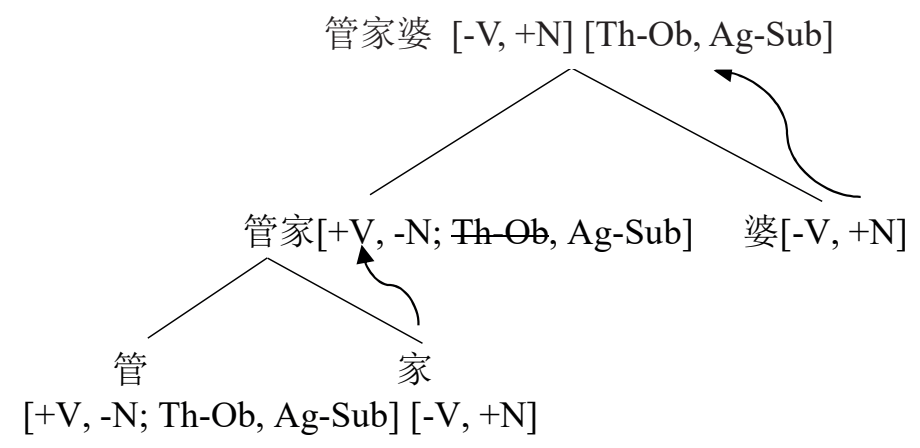

Die Struktur in (13) unterscheidet sich von (10) lediglich durch die Stellung des Objekts, das sich in (13) rechts vom Verb befindet. Ansonsten gleicht die Argumentvererbung der in (10), was die entsprechenden Pfeile und Durchstreichungen symbolisieren. Nach diesem Muster werden z. B. folgende RKs gebildet: 参保人员 (can bao renyuan -, Versicherter'), 办案人 (ban an ren -,Ermittler'), 领军人 (ling jun rencai -,Spitzenreiter').

Erwähnenswert ist auch ein weiterer Typ von RKs, in denen das Verb äußerlich betrachtet zwei Objekte bei sich hat, zum Beispiel in 新闻播音员 (xinwen boyin yuan ,Nachrichtensprecher'). Die komplexe Konstituente in der Mitte 播音 (boyin - ,senden') besteht aus zwei Teilen: 播 und 音. 播 ist der grundlegende Verbstamm und 音 ist sein Objekt. Anscheinend verfügt das Verb über zwei direkte Objekte, was z. B. alleine aufgrund der Thetatheorie nicht möglich sein dürfte. Dies lässt sich aber so erklären, dass das Verb sein erstes Objekt „inkorporiert“, dieses sättigt keine Argumentstelle, infolgedessen eine komplexe, kategorial verbale Wortstruktur entsteht, die fähig ist, ein weiteres Objekt anzuschließen. Exemplarisch sieht die Analyse wie folgt aus:
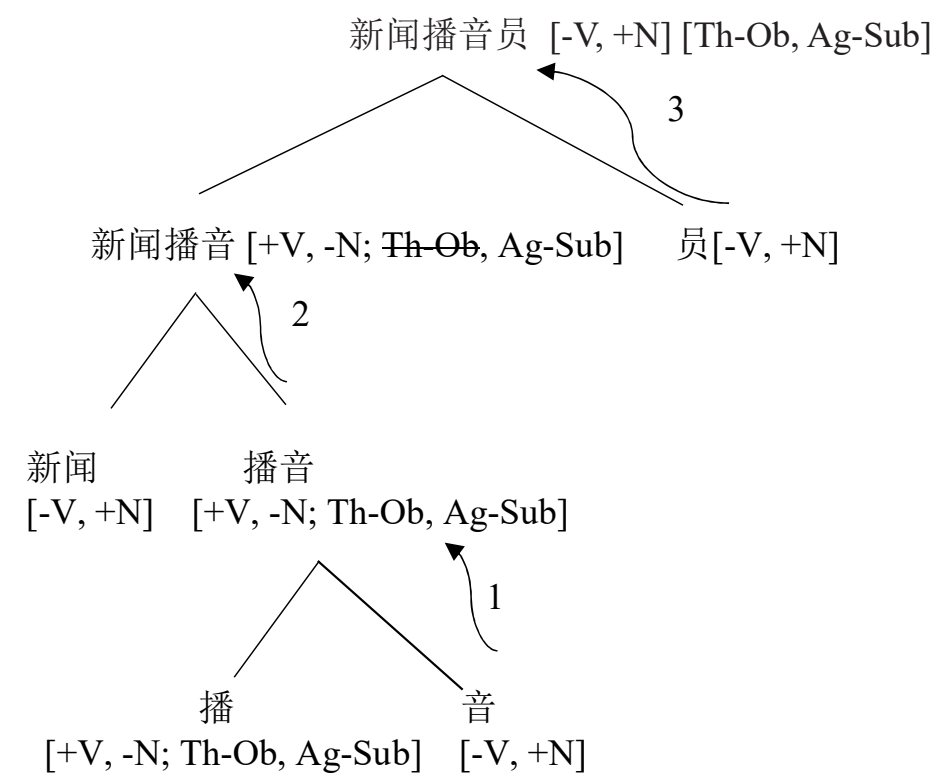
Auf Stufe 1 inkorporiert der Verbstamm die erste nominale Konstituente, wodurch jedoch keine Argumentstelle gefüllt wird. Ein solches komplexes Verb geht danach auf Stufe 2 eine Rektionsbeziehung mit dem Objekt 新闻 (xinwen - ,Nachricht ${ }^{6}$ ) ein, wodurch die Objektrolle realisiert wird (Durchstreichung). Schließlich wird das Subjekt (员 - yuan), das hier den Konstruktionskopf bildet, angeschlossen, womit die Bildung des RK abgeschlossen ist.

\section{Zusammenfassung}

In den beiden Sprachen sind RKs als Nomina agentis rechtsköpfig, ihr Kopf ist das Subjekt das Basisverbs, im Deutschen wird es als ein über eine allgemeine Bedeutung verfügendes Suffix realisiert, im Chinesischen dagegen als ein Morphem, das ein menschliches (geschlechtsneutrales) Wesen bezeichnet, reihenbildend auftritt und seine Sachbedeutung weitgehend behalten hat. Unterschiede zwischen dem Deutschen und dem Chinesischen betreffen die Abfolge der OV-Konstituenten: Im Deutschen hat man es mit der festen Anordnung OV zu tun, im Chinesischen sowohl mit OV als auch mit VO, was in der Folge auch abweichende Ableitungsmechanismen voraussetzt. Nomina actionis im Deutschen lassen sich aufgrund suffixaler Exponenten leicht identifizieren, während dies im Chinesischen in Ermangelung solcher Exponenten nicht möglich ist.

Die präsentierte Darstellung versteht sich als Beitrag zur Erforschung eines äußerst interessanten Ausschnitts der Wortbildung, wobei die vergleichende Perspektive tiefere Einsichten in die Derivationsmechanismen der RKs in der jeweils gegenübergestellten Sprache gewinnen ließ. Da man es hier mit genetisch wie typologisch verschiedenen Sprachen zu tun hat, können Ergebnisse allgemeinlinguistisch verwertet werden.

\section{Literatur}

Aptacy, J. (2015a). Ein lesenswerter und korrekturfreundlicher Aufsatz. Zu internen Beziehungen zwischen Konstituenten adjektivischer Rektionskomposita im Deutschen. Studia Linguistica, 34, 7-22.

Aptacy, J. (2015b). Rektionskomposita und Argumentvererbung - ein deutsch-polnischer Vergleich. Zeitschrift des Verbandes Polnischer Germanisten, H.1, 1-16. Abgerufen von http://www.ejournals.eu/ ZVPG/2015/Zeszyt-1/art/4766/

Bierwisch, M. (1989). Event Nominalization: Proposals and Problems. In W. Motsch (Hrsg.), Wortstruktur und Satzstruktur (S. 1-73). Berlin: Akademie der Wissenschaften der DDR.

Cheng, G. (2005). Zhe (er) synthetic compounds in Chinese and their implications for UG. Modern Foreign Languages, 28, 232-238.

Eichinger, L.M. (2000). Deutsche Wortbildung. Eine Einführung. Tübingen: Narr.

Fanselow, G. (1981). Neues von der Kompositafront oder Zu drei Paradigmen in der Kompositagrammatik. Studium Linguistik, 11, 43-57.

Fleischer, W. \& Barz, I (2012). Wortbildung der deutschen Gegenwartssprache. Berlin-Boston: de Gruyter. 
Fuhrhop, N. (2007). Zwischen Wort und Syntagma. Zur grammatischen Fundierung der Getrennt- und Zusammenschreibung. Tübingen: Niemeyer.

Greenberg, J.H. (1963). Some Universals of Grammar with Particular Reference to the Order of Meaningful Elements. In J. Greenberg (Hrsg.), Universals of Language (S. 73-113). Cambridge (Mass.): MIT Press. Gu, Y. \& Shen, Y. (2001). The derivation of synthetic compounds in Chinese. Zhongguo Yuwen, 2, 122-133. Haspelmath, M. \& Sims, A.D. (2010). Understanding Morphology. London: Hodder Education.

He, Y. (2004). The loop theory in Chinese morphology. Dangdai Yuyanxue, 6, 223-235.

He, Y. (2013). A further study on Chinese synthetic compounds: Structure, typology and cues to language acquisition. Foreign Language Teaching and Research, 45, 483-494.

Hong, S. \& Shi, D. (2012). On Construction of Chinese Compounds. TCSOL Studies, 4, 76-80.

Kürschner, W. (1974). Zur syntaktischen Beschreibung deutscher Nominalkomposita. Tübingen: Niemeyer.

Lieber, R. (1983). Argument Linking and Compounds in English. Linguistic Inquiry, 14, 251-285.

Olsen, S. (1986a). „Argument-Linking“ und unproduktive Reihen bei deutschen Adjektivkomposita. Zeitschrift für Sprachwissenschaft, 5 (1), 5-24.

Olsen, S. (1986b). Wortbildung im Deutschen. Eine Einführung in die Theorie der Wortstruktur. Stuttgart: Kröner.

Rivet, A. (1999). Rektionskomposita und Inkorporationstheorie. Linguistische Berichte, 179, 307-342.

Sabel, J. (2002). Die Doppelobjekt-Konstruktion im Deutschen. Linguistische Berichte, 190, 229-244.

Scalise, S. \& Vogel, I. (2010). Why compounding? In I. Vogel \& S. Scalise (Hrsg.), Cross-disciplinary Issues in Compounding (S. 1-18). Amsterdam: John Benjamins.

Schmidt, G.D. (1987). Das Affixoid. Zur Notwendigkeit und Brauchbarkeit eines beliebten Zwischenbegriffs der Wortbildung. In G. Hoppe et al. (Hrsg.), Deutsche Lehnwortbildung. Beiträge zur Erforschung der Wortbildung mit entlehnten WB-Einheiten im Deutschen (S. 53-101). Tübingen: Narr.

Szigeti, I. (2002). Nominalisierungen und Argumentvererbung im Deutschen und Ungarischen. Tübingen: Niemeyer.

Toman, J. (1983). Wortsyntax. Eine Diskussion ausgewählter Probleme deutscher Wortbildung. Tübingen: Niemeyer.

Vater, H. (2002). Einführung in die Sprachwissenschaft. München: Fink.

Zhuang, H. \& Liu, Z. (2011). Morphology of Chinese Synthetic Compounds and Their Prosodic Constraint. Shijie Hanyu Jiaoxue, 25, 497-506. 